\title{
Study of Polarized Wave with a Hydrodynamic Model and Fourier Spectral Method
}

\author{
Dennis Ling Chuan Ching, ${ }^{1}$ Zainal Abdul Aziz, ${ }^{2}$ and Faisal Salah ${ }^{3}$ \\ ${ }^{1}$ Department of Fundamental and Applied Science, Universiti Teknologi PETRONAS, 31750 Tronoh, Perak, Malaysia \\ ${ }^{2}$ UTM Centre for Industrial and Applied Mathematics, Department of Mathematical Sciences, Faculty of Science, \\ Universiti Teknologi Malaysia (UTM), 81310 Johor Bahru, Malaysia \\ ${ }^{3}$ Department of Mathematics, Faculty of Science, University of Kordofan, North Kordofan State, El-Obeid 51111, Sudan
}

Correspondence should be addressed to Dennis Ling Chuan Ching; dennislingcc@gmail.com

Received 2 September 2013; Revised 25 November 2013; Accepted 5 December 2013

Academic Editor: ShengKai Yu

Copyright (C) 2013 Dennis Ling Chuan Ching et al. This is an open access article distributed under the Creative Commons Attribution License, which permits unrestricted use, distribution, and reproduction in any medium, provided the original work is properly cited.

The polarization effects in hydrodynamics are studied. Hydrodynamic equation for the nonlinear wave is used along with the polarized nonlinear waves and seismic waves act as initial waves. The model is then solved by Fourier spectral and Runge-Kutta 4 methods, and the surface plot is drawn. The output demonstrates the inundation behaviors. Consequently, the polarized seismic waves along with the polarized nonlinear waves tend to generate dissimilar inundation which is more disastrous.

\section{Introduction}

Via the massive energy released by the faults, energy of the earthquake is usually measured in magnitude or Rachel scale. Released energy in less than one percent of is said transmitted to form Tsunamis [1]. Therefore, we assume that the Tsunami and seismic waves should exist together in this paper during the derivation of polarized model. The notion is strong ground motion by the wave field polarization that generates Tsunami. Reflection being transmitted or polarization gives strong earth motion. Seismic waves due to strong polarized earthquakes propagating in seabed layers will reduce soil stiffness and increase the energy dissipation into the soil $[2,3]$.

Lately, recording of polarization of the Earth's teleseismic wave fields and strong ground motion is imaged by applying a vector stacking method to three component broadband seismograms [4]. Polarization effect of the $\mathrm{P}$ waves and $\mathrm{S}$ waves was also studied analytically for the dispersion and attenuation effects in fluid saturated medium [5-8].

Besides, wave field polarization may both amplify the displacement and increase the attenuation. It is found that multiple wave fields impinging each other give amplification effect while wave field separation gives the negative damping effects [9]. By considering the polarization of seismic wave to the generation of water displacement, we analyze the effect of strong polarized seabed motion towards the generation of Tsunamis.

Tsunamis are divided into breaking and nonbreaking waves. Analytical approach is used to study the behavior of Tsunamis theoretically [10-13]. Zelt [14] used Boussinesq equations for deriving breaking and nonbreaking Tsunami run-ups.

There are experimental approaches for exploring nonlinear wave's motion [15-18]. Experimental results by Hwang et al. [19] indicated that the Tsunamis run-ups are directly proportional to the incline beach plane and the run-ups are higher than the run-ups height proposed by Synolakis [13], and Hwang et al. [19] had conducted a study in water tank with $1 / 20$ beach plane. Chang and Hwung [17] and Hsiao et al. [18] implemented water tank experiments for wave propagation in a water tank $(300 \mathrm{~m} \times 5 \mathrm{~m} \times 5.2 \mathrm{~m})$ with incline beach planes $1 / 40$ and $1 / 60$ at Tainan Hydraulics Laboratory of National Cheng Kung University. Besides, the 
wave flume $(2 \mathrm{~m} \times 2 \mathrm{~m} \times 200 \mathrm{~m})$ is as well implemented by Chang and Hwung [17] with the purpose of reducing the scale effect.

There are approaches to modeling run-up on beach: the nonlinear shallow water equations (nondispersive system) and Boussinesq equations to include the dispersion in weakly nonlinear waves. More sophisticated methods such as NavierStokes equations are used to simulate wave run-up, that is, UNESCO TSUNAMI model [20]. The tsunami model TsunAWI by Alfred Wegener Institute simulates all stages of a tsunami from the origin and the propagation in the ocean to the arrival at the coast and the inundation on land. Solved nonlinear shallow water equations on an unstructured finite element grid allows changing the resolution seamlessly between a coarse grid in the deep ocean and a fine representation of coastal structures. Besides, it provides fast computation [21].

Here, we use the dimensionless equation of hydrodynamics (1) which is derived in accordance with Boussinesq equations by Thierry and Michel [22]:

$$
\frac{\partial u}{\partial t}+\frac{\partial u}{\partial x}+\frac{3}{2} u \frac{\partial u}{\partial x}+\frac{1}{6} \frac{\partial^{3} u}{\partial x^{3}}=0,
$$

in which the dispersion term $u_{x x x}$ represents shock formation produced by convective term. $u u_{x}$ represents the steepening of the wave while $u_{x x x}$ represents the depressiveness of the wave. The last phase of a tsunami is its run-ups and inundation. With linear speed $c_{0}=\sqrt{g h}$, dimensionless variables are

$$
x \equiv \frac{\bar{x}}{l}, \quad t=\frac{\bar{t}}{l / c_{0}}, \quad u=\frac{\bar{u}}{l c_{0}} .
$$

\section{Polarization}

However, a simple calculation based on considering the tsunami has a wave profile that reads [23]

$$
u_{0}=2 \operatorname{sech}^{2}(x)
$$

given for the $x$-axis direction propagation only which is not right to explain the waves propagates in the $x-z$ directions or run-ups at the incline beach plane.

Figures 1(a) and 1(b) illustrate the difference between constant amplitude and variable amplitude for nonlinear waves. The conventional wave (3) that is used in modelling is shown in Figure 1(a). Figure 1(b) illustrates the schematic model of this research. The nonlinear wave is variable because of the reflection at beach plane. Incidence of seismic plane wave is not left behind whereas both the nonlinear waves and seismic waves make a polarization angle $\nu$ during the Tsunami run-ups.

Complying with the Snell laws, the variation of density will lead to the seismic wave diffraction during incidence. Here, the diffraction is due to polarization and consequently the purposed objective is to derive the polarized nonlinear waves and seismic waves as initial waves in hydrodynamics simulation. In next session, the derivation for polarization is introduced before the application of polarization in the nonlinear waves and the seismic waves. Then, numerical simulation and discussion will be shown.

In Figure 1, $C$ is the incident wave coefficient with $A=$ $A(x, z)$ and $M$ is the coefficients for wave height variation $A \partial z$ and distance travelled $M \partial x$. Initial wave height $A(x, z)$ with minimal variation $\delta z$ reads

$$
A(x, z) \delta z \cong A(x, 0) \delta z=A(x) \delta z .
$$

The wave height depends on the distance travelled or $A=$ $A(x)$. Essentially, we are going to find the polarization angle $v$ from total variation angle $\theta^{\prime}$. In accordance with Figure 1, shock dynamics relations with the angle $\theta(x, z)$ between the $x$-axis and the ray were derived:

$$
\begin{gathered}
\frac{\delta \theta}{\delta z}=\frac{(A+(\partial A / \partial x) \delta x) \delta z-A \delta z}{M \delta x}=\frac{1}{M} \frac{\partial A}{\partial x} \\
\frac{\delta \theta}{\delta x}=\frac{M \delta x-(M+(\partial M / \partial z) \delta z) \delta x}{A \delta z}=-\frac{1}{A} \frac{\partial M}{\partial z} .
\end{gathered}
$$

In this section, we will show the derivation for polarization along with the angle between the beach plane and $x$-axis $\theta(x, z)$ and polarization angle $v$ according to Han and Yin [24]. The total variation angle $\theta^{\prime}$ is

$$
\theta^{\prime}=\theta+\nu
$$

For the wave orientation, it is appropriate to write the variation as

$$
\frac{\delta \theta}{\delta z}=\frac{\partial \theta}{\partial z}=w(x, z), \quad \frac{\delta \theta}{\delta x}=\frac{\partial \theta}{\partial x}=u(x, z) .
$$

Respectively, $u$ and $w$ are the variation in horizontal and vertical direction. Hence, relations (5) become

$$
\begin{gathered}
\frac{\partial \theta}{\partial z}=\frac{1}{M} \frac{\partial A}{\partial x}, \\
\frac{\partial \theta}{\partial x}=-\frac{1}{A} \frac{\partial M}{\partial z} .
\end{gathered}
$$

Subsequently, the polarization angle $v$ will be extracted from the (8a) and (8b), given that $\theta^{\prime}=\theta+\nu$.

By inserting $A=A(M)$ into (8a), the relations read

$$
\begin{gathered}
\frac{\partial \theta}{\partial x}+\frac{1}{A} \frac{\partial M}{\partial z}=0 \\
\frac{\partial \theta}{\partial z}-\frac{A^{\prime}}{M} \frac{\partial M}{\partial x}=0 \quad \text { with } A^{\prime}=\frac{d A}{d M}
\end{gathered}
$$

Equations (9a) and (9b) can be written in characteristic form. By introducing $(9 a) \pm C \times(9 b)$ with $C$ being the coefficient of incident waves, one gets the relations of variation in characteristic form:

$$
\begin{aligned}
& \left(\frac{\partial}{\partial x}+C \frac{\partial}{\partial z}\right)\left(\theta+\frac{M}{A C}\right)=0 \\
& \left(\frac{\partial}{\partial x}-C \frac{\partial}{\partial z}\right)\left(\theta-\frac{M}{A C}\right)=0 .
\end{aligned}
$$




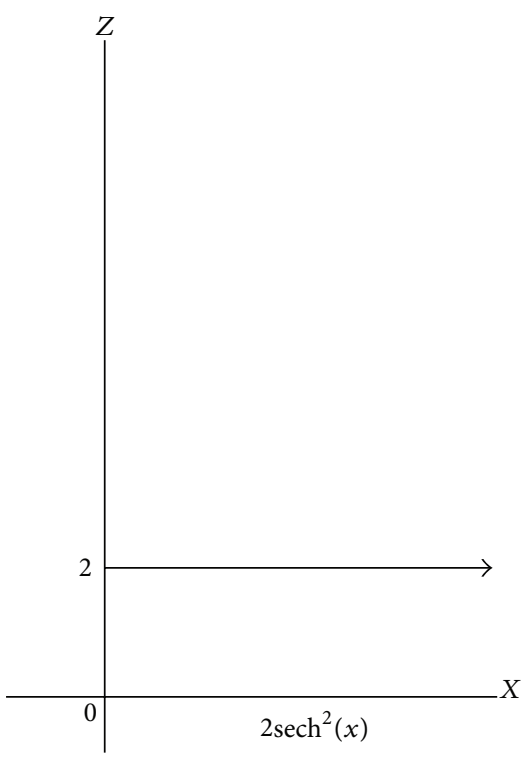

(a)

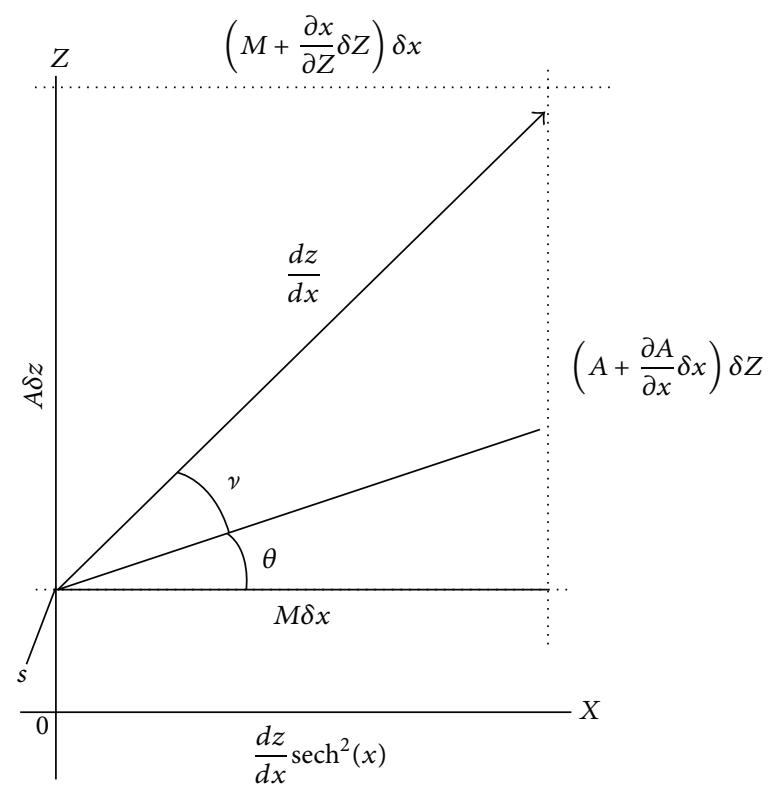

(b)

FIgURE 1: The nonlinear waves. (a) The nonlinear wave propagates with amplitude 2 in $x$-direction. (b) The nonlinear waves propagate with variation amplitude $d z / d x$, initiated by reflection at incline beach plane. There is an incidence of seismic plane waves, $s$ from the seabed whereas the seismic plane waves suffer diffraction initially.

Equations (10a) and (10b) will limit minimum beach plane gradient to $\theta=1$ such that

$$
\frac{M}{A C} \neq 1
$$

The characteristic curves of incident waves, $C$ and Riemann invariants $r$ and $s$ are

$$
\begin{array}{ll}
C^{+}=\frac{d z}{d x}, & r=\theta+\frac{M}{A C}, \\
C^{-}=\frac{d z}{d x}, & s=\theta-\frac{M}{A C} .
\end{array}
$$

Consequently, the total variation angle is

$$
\theta^{\prime}=\theta \pm v \quad \text { with } \nu=\frac{M}{A C}
$$

From (10a) and (10b), it is noted that

$$
\frac{M}{A C}>1
$$

The value for $v>1$ will be used for the numerical simulation later. By solving (10a) and (10b), the total variation due to polarization reads

$$
\frac{d z}{d x}=\tan (\theta+v) \quad \text { with } C=\sqrt{\left(\frac{\partial M}{\partial z}\right)^{2}+\left(\frac{\partial A}{\partial x}\right)^{2}} .
$$

Next, the derivation of initial waves with polarization will be explained.

\section{Initial Waves}

In this modelling, we assume that the nonlinear waves and the seismic plane waves will share single polarization angle $v$. For the cases of multiple polarization angle $v$, it will be studied in further research.

For deriving the polarized nonlinear waves, the amplitude in (3) is replaced with $d z / d x$ or $\tan (\theta+\nu)$ as illustrated in Figure 1(b). The initial waves read

$$
u=\tan (\theta+v) \operatorname{sech}^{2}(x-c t)
$$

and the polarized seismic waves are

$$
u=\tan (\theta+v) \exp [i k(c t-x)]
$$

where $c$ is the phase velocity of the wave. We are assuming that the initial seismic waves are plane waves with the amplitude depending on the polarization or $d z / d x$. Both (17) and (18) are used as initial waves in this analysis in conjunction with the hydrodynamics equation (1). Fourier spectral method and Runge-Kutta 4 methods are used to solve the identified model.

\section{Fourier Spectral and Runge-Kutta 4 Methods}

Hydrodynamics equation (1) is rewritten as

$$
u_{t}+u_{x}+\frac{3}{2}\left(\frac{1}{2} u^{2}\right)_{x}+\frac{1}{6} u_{x x x}=0 .
$$




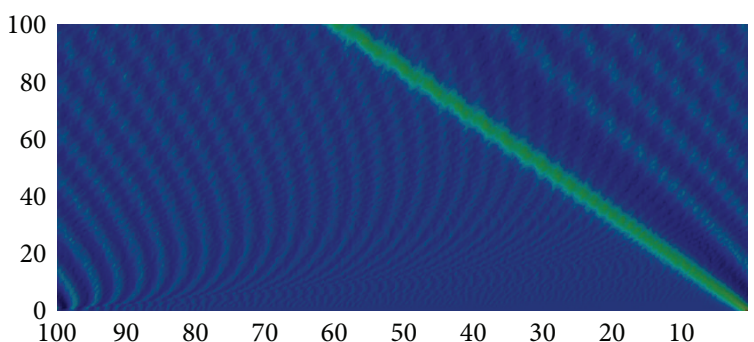

(a) $[0,32 \mathrm{pi}]$

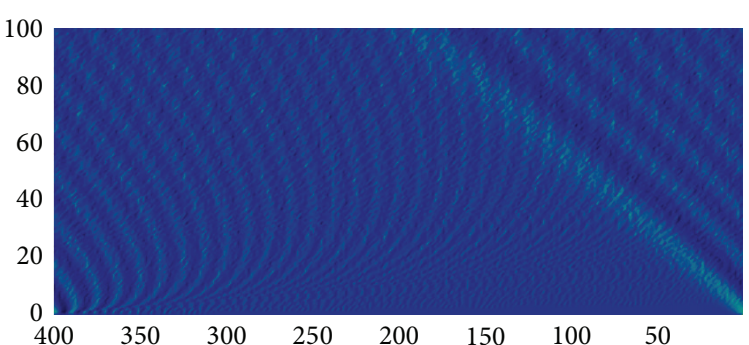

(b) $[0,128 \mathrm{pi}]$

FIGURE 2: Time evolution for the hydrodynamics equation together with initial wave $u=2 \operatorname{sech}^{2}(x)$. Time runs from 0 at the bottom of the figure to 100 at the top.

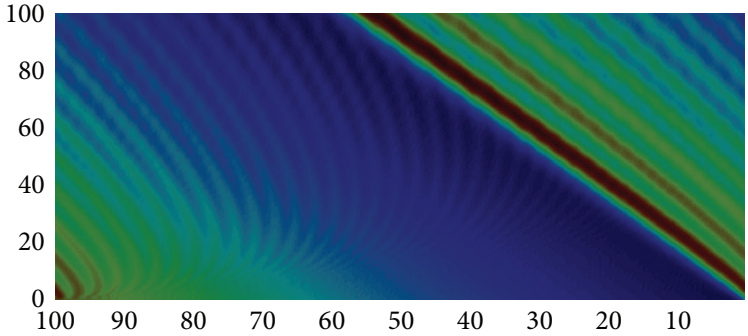

(a) $[0,32 \mathrm{pi}]$

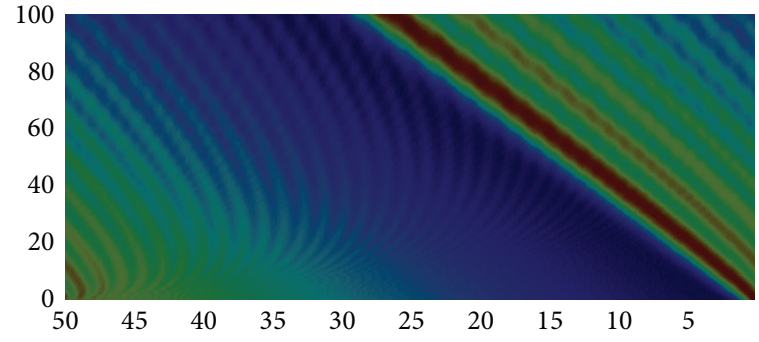

(b) $[0,16 \mathrm{pi}]$

FIGURE 3: Time evolution for the hydrodynamics equation together with initial wave $u=\tan (\theta) \operatorname{sech}^{2}(x-c t)+\tan (\theta) \exp [i k(x-c t)], \theta=0.1$ and $v=0$.

Spatial part of hydrodynamics equation will be discretized by using Fourier spectral method. The Fourier space gives

$$
\widehat{u}_{t}=-\frac{i k}{2} \widehat{u}^{2}+\left(i k^{3}-i k\right) \widehat{u}
$$

or, in standard form of $u_{t}=L u+N(u, t)$,

$$
\begin{gathered}
(L \widehat{u})(k)=\left(i k^{3}-i k\right) \widehat{u}(k), \\
N(\widehat{u}, t)=N(\widehat{u})=-\frac{i k}{2}\left(F\left(\left(F^{-1}(\widehat{u})\right)^{2}\right)\right),
\end{gathered}
$$

where $L$ and $N$ are the linear and nonlinear operators, respectively. Then, we use Runge-Kutta 4 for time stepping. Initial waves from (17) and (18) will be used to analyze the effect of polarization.

\section{Results and Discussions}

In this section, the configurations of hydrodynamics models as shown in Figures 1(a) and 1(b) will be shown. The initial waves of (3) will produce the initial waves with dimensionless amplitude 2. Figure 2 shows the initial waves generated for two different domains: (a) [0,32pi], (b) [0, 128pi]. Time runs from 0 at the bottom of the Figure to 100 at the top and the initial wave's propagation began from the right of both Figures 2(a) and 2(b).

From the Figures 2(a) and 2(b), stability of the initial waves is shown to be greatly influenced by the size of the domain; the reflected waves tend to diminish the waves when the size of the domain increases as shown in Figure 2(b). However, the dispersion of the waves is still significant in both Figures 2(a) and 2(b). There is no beach plane or run-ups in Figures 2(a) and 2(b) since the initial waves (3) with constant amplitude are being used.

Subsequently, the consequences of the Tsunami run-ups at the beach plane are shown without the polarization angle, $\nu$. The initial waves are derived with $v=0$ such that the initial waves read

$$
u=\tan (\theta) \operatorname{sech}^{2}(x-c t)+\tan (\theta) \exp [i k(c t-x)]
$$

Figures 3(a) and 3(b) are plotted to show the run-ups by initial waves (22).

For these flow cases, we should see a beach plane when $t=0$ as shown in Figures 3(a) and 3(b). Figures 3(a) and 3(b) demonstrate the time evolution of the wave's run-ups without polarization by letting $\nu=0$. The angle between beach plane and $x$-axis is $\theta=0.1$.

Consequently, dispersion is reduced when the size of the domain increases. By comparing Figures 3(a) and 3(b), the dispersive waves are more significant in Figure 3(a). Nevertheless, the initial wave comes with the displacement height around 1.2 units which is higher than the initial wave as shown in Figure 3(b) with the displacement height around 0.33 in dimensionless. When the length of the wave is evaluated, the wave shown in Figure 3(b) is found longer. Apparently, the weak dispersion has led to the long waves in Figure 3(b). 


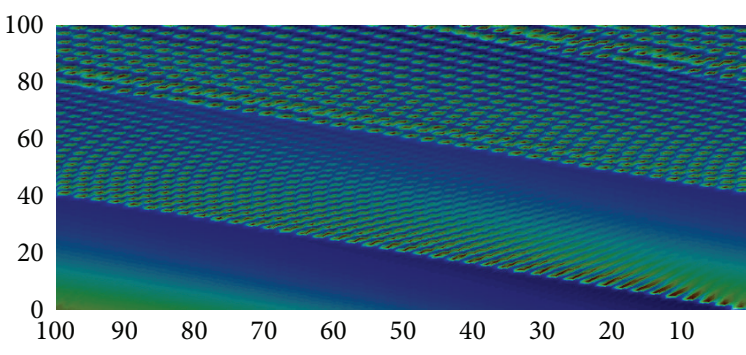

(a) $[0,32 \mathrm{pi}]$

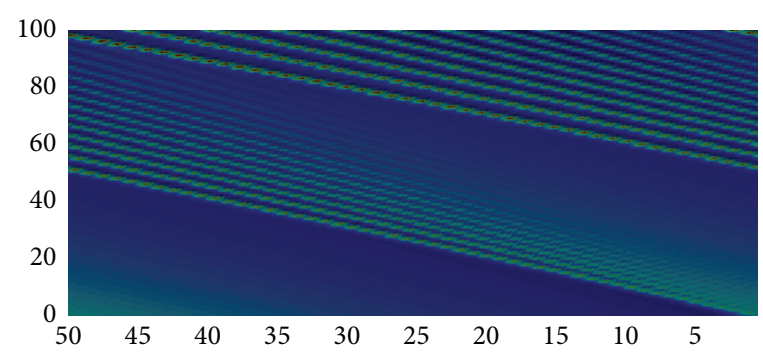

(b) $[0,16 \mathrm{pi}]$

FIGURE 4: Time evolution for the hydrodynamics equation together with initial wave $u=\tan (\theta+\nu) \operatorname{sech}^{2}(x-c t)+\tan (\theta+\nu) \exp [i k(x-c t)]$, $\theta=0.1$ and $v=1$

Inundation comes in after time $t=20$ for domain [0, $32 \mathrm{pi}]$ and $t=70$ for domain [0,16pi]. The inundation comes in earlier when the domain is larger for which we presumed the consequence being brought forward by the quicker pace of initial waves by referring to the simple velocity equation, $x / t$. Longer domain $x$ with similar amount of time evolution will generate greater velocity. This incidence is proven in Figures 3(a) and 3(b).

However, the reflected waves are still visible. In Figures 2(a) and 2(b), the reflected waves are shown to diminish the incoming waves when the domain increases. In contrast, the incoming wave run-ups on beach plane without the polarization effect are greater and higher in displacement when the domain increases as shown in Figures 3(a) and 3(b).

The nonpolarized initial waves were shown previously. Here, the consequence of polarized initial waves in hydrodynamics will be shown. Figures 4(a) and 4(b) illustrate the polarized initial waves with $\theta=0.1$ and $\nu=1$. Similarly, the initial waves are simulated in two domains: (a) [0, 32pi] and (b) $[0,16 \mathrm{pi}]$.

Apparently, the Figures 4(a) and 4(b) show dissimilar illustration as Figures 2 and 3, respectively. The initial waves when $t=0$ show extraordinary initial displacement with displacement height around 7 in dimensionless as shown in Figure 4(a). Nevertheless, the displacement height is around 6 units as shown in Figure 4(b). These high displacements have totally ignored the existence of reflected waves.

By comparing the inundation time of Figure 3(a) with Figure 4(a), the paces of the initial waves are quicker. This indicates that the velocity of the waves is higher. The initial waves arrive at the shore or $x=100$ when $t=40$ units. When the domain reduces, the arrival time of the waves at the shore is reduced as well. Figure 4(b) shows the inundation time at $t=50$.

The polarization effect in Tsunami wave is referred to the situation for the near-shore Tsunami. From the source of the earthquake, the Indian Ocean Tsunami is known as near-shore Tsunami. If the polarization effect is ignored, the inundation in Sumatra in 2004 is less disastrous as shown in Figure 3(b). However, the inundation is deeper and comes in earlier once the polarization effect is considered as shown in Figure 4(b).

\section{Conclusion}

The development of hydrodynamics model with the existence of seismic waves is a promising task. Type of setup especially the structure of the bottom geography is important. The nonlinear wave in the domain without an inclined beach plane is responsive to the reflected waves. However, an incline beach plane will show no reflected waves when the polarization in waves is considered.

The size of the domain denotes the roles of polarization. The Tsunami wave propagates in the smaller domain exclusive of polarization effect is longer in size, but the inundation is less profound once compared with the Tsunami wave embedded with polarization. With the polarization effects, the tsunami wave is catastrophic: the height of the displacement is greater than before and the inundation comes earlier and deeper for which we take for granted the hazard is being brought forward by the high displacement.

\section{Conflict of Interests}

The authors declare that there is no conflict of interests regarding the publication of this paper.

\section{Acknowledgment}

The authors will like to thank UTP for the financial support.

\section{References}

[1] T. Lay, H. Kanamori, C. J. Ammon et al., "The great SumatraAndaman earthquake of 26 December 2004," Science, vol. 308, no. 5725, pp. 1127-1133, 2005.

[2] M. P. S. d'Avila, L. Lenti, and J.-F. Semblat, "Modelling strong seismic ground motion: three-dimensional loading path versus wavefield polarization," Geophysical Journal International, vol. 190, no. 3, pp. 1607-1624, 2002.

[3] P. S. Earle, "Polarization of the Earth's teleseismic wavefield," Geophysical Journal International, vol. 139, no. 1, pp. 1-8, 1999.

[4] M. D. Sharma, "Effect of initial stress on reflection at the free surface of anisotropic elastic medium," Journal of Earth System Science, vol. 116, no. 6, pp. 537-551, 2007. 
[5] Z. A. Aziz, L. Dennis, and S. Y. Faisal, "Scattering of SH-waves in fluid-saturated medium," Applied Mathematical Sciences, vol. 4, no. 68, pp. 3375-3386, 2010.

[6] Z. A. Aziz, L. Dennis, and S. Y. Faisal, "Scattering of SV waves in fluid-saturated medium," Australian Journal of Basic and Applied Sciences, vol. 4, no. 8, pp. 3843-3853, 2010.

[7] L. Dennis, S. Y. Faisal, and Z. A. Aziz, "P-waves diffusion in fluid-saturated medium," Matematika, vol. 26, no. 1, pp. 53-60, 2010.

[8] L. Dennis, S. Y. Faisal, and Z. A. Aziz, "SH waves diffusion in fluid-saturated medium," Journal of Mathematics and Statistics, vol. 6, no. 3, pp. 205-209, 2010.

[9] D. Donno, A. Nehorai, and U. Spagnolini, "Seismic velocity and polarization estimation for wavefield separation," IEEE Transactions on Signal Processing, vol. 56, no. 10 I, pp. 47944809, 2008

[10] G. F. Carrier, T. T. Wu, and H. Yeh, "Tsunami run-up and drawdown on a plane beach," Journal of Fluid Mechanics, no. 475, pp. 79-99, 2003.

[11] U. Kânoǧlu and C. E. Synolakis, "Long wave runup on piecewise linear topographies," Journal of Fluid Mechanics, vol. 374, pp. 128, 1998.

[12] U. Kânoğlu, "Nonlinear evolution and runup-rundown of long waves over a sloping beach," Journal of Fluid Mechanics, vol. 513, pp. 363-372, 2004.

[13] C. E. Synolakis, "Tsunami runup on steep slopes: how good linear theory really is," Natural Hazards, vol. 4, no. 2, pp. 221234, 1991.

[14] J. A. Zelt, "The run-up of nonbreaking and breaking solitary waves," Coastal Engineering, vol. 15, no. 3, pp. 205-246, 1991.

[15] J. V. Hall and J. W. Watts, "Laboratory investigation of the vertical rise of nonlinear waves on impermeable slopes," Beach Erosion Board Tech. Memo. 33, US Army Corps of Engineers, Vicksburg, Miss, USA, 1953.

[16] Y. Li and F. Raichlen, "Non-breaking and breaking solitary wave run-up," Journal of Fluid Mechanics, vol. 456, pp. 295-318, 2002.

[17] Y. H. Chang and H. H. Hwung, "Experiments on the run-up of nonlinear Waves over sloping bottoms," in Proceedings of the 3rd Chinese-German Joint Symposium on Coastal and Ocean Engineering, National Cheng Kung University, Tainan, Taiwan, 2006.

[18] S. C. Hsiao, T. W. Hsu, T. C. Lin, and Y. H. Chang, "On the evolution and run-up of breaking solitary waves on a mild sloping beach," Coastal Engineering, vol. 55, no. 12, pp. 975-988, 2008.

[19] K. S. Hwang, H. H. Hwung, H. H. Chen, H. Y. Huang, and Y. H. Chang, "Large scale experiments on the run-up of nonlinear waves on 1:20 slope," in Proceedings of the 27th Ocean Engineering Conference in Taiwan, National Chung Hsian University, Chiayi County, Taiwan, 2005.

[20] IUGG/IOC TIME Project, Numerical Method of Tsunami Simulation with the Leap-Frog Scheme, vol. 35 of Manuals and Guides, Intergovernmental Oceanographic Commission, 1997.

[21] N. Rakowsky, A. Androsov, A. Fuchs et al., "Operational tsunami modelling with TsunAWI-recent developments and applications," Natural Hazards and Earth System Sciences, vol. 13, pp. 1629-1642.

[22] D. Thierry and P. Michel, Physics of Solitons, Cambridge University Press, New York, NY, USA, 2006.

[23] M. W. Dingemans, Wave Propagation over Uneven Bottoms, vol. 13 of Advanced Series on Ocean Engineering, World Scientific, Singapore, 1997.
[24] Z. Y. Han and X. Z. Yin, Shock Dynamics, vol. 11 of Fluid Mechanics and Its Applications, Kluwer Academic, Amsterdam, The Netherland, 1993. 

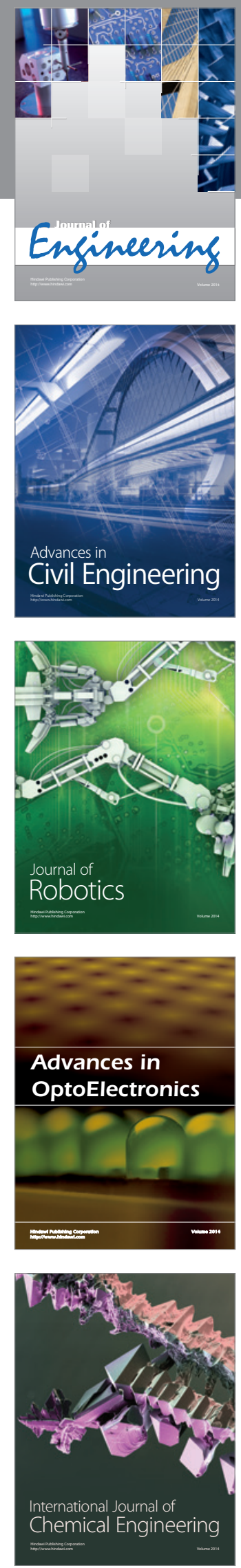

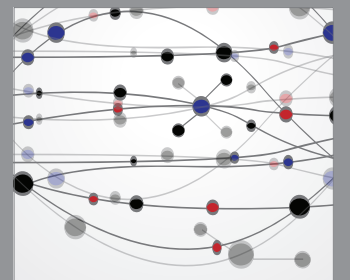

The Scientific World Journal
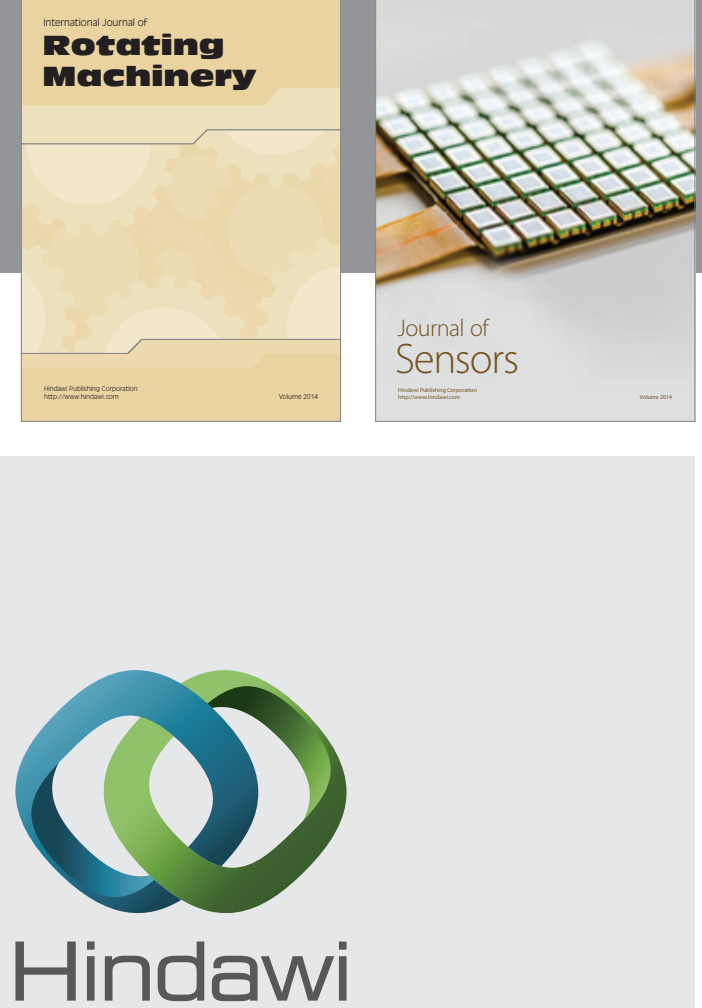

Submit your manuscripts at http://www.hindawi.com
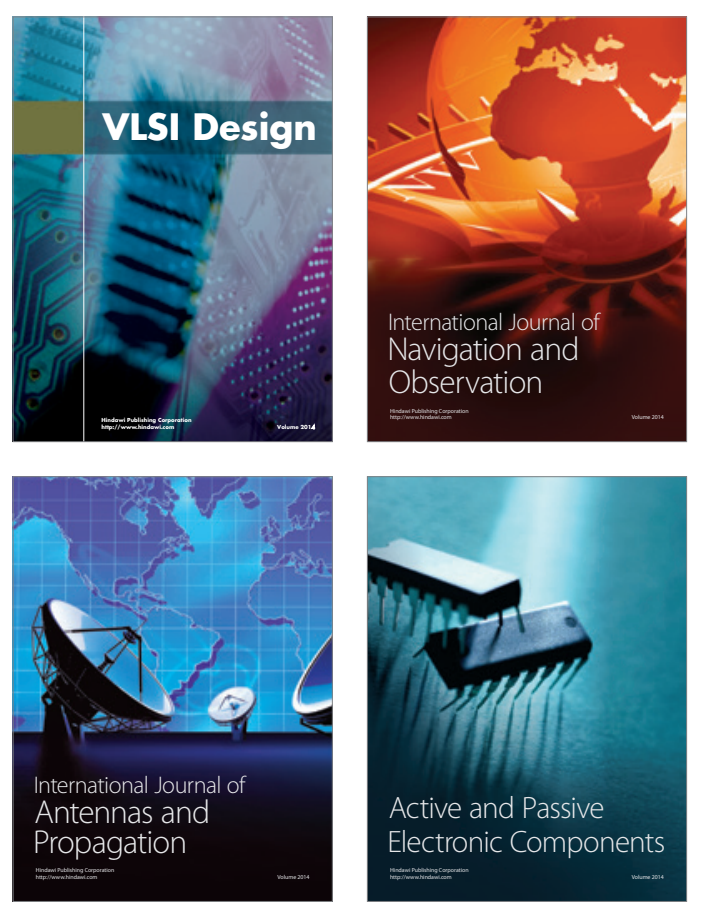
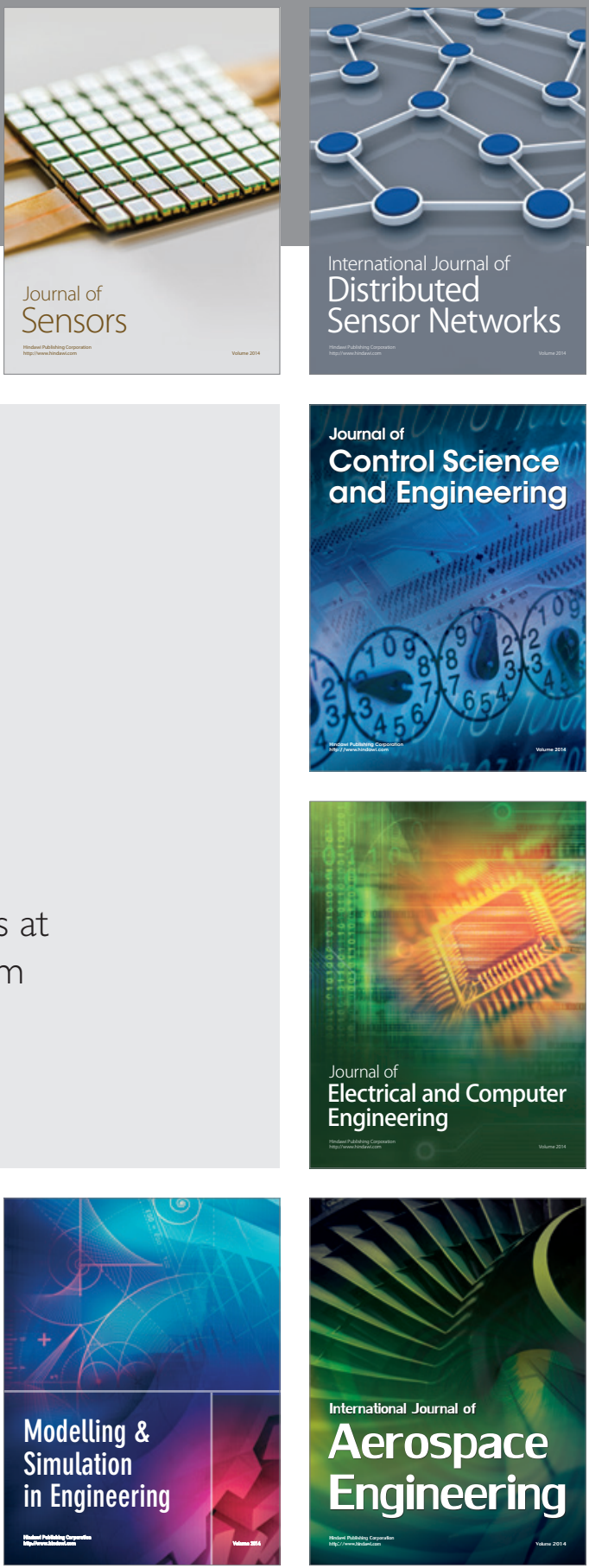

Journal of

Control Science

and Engineering
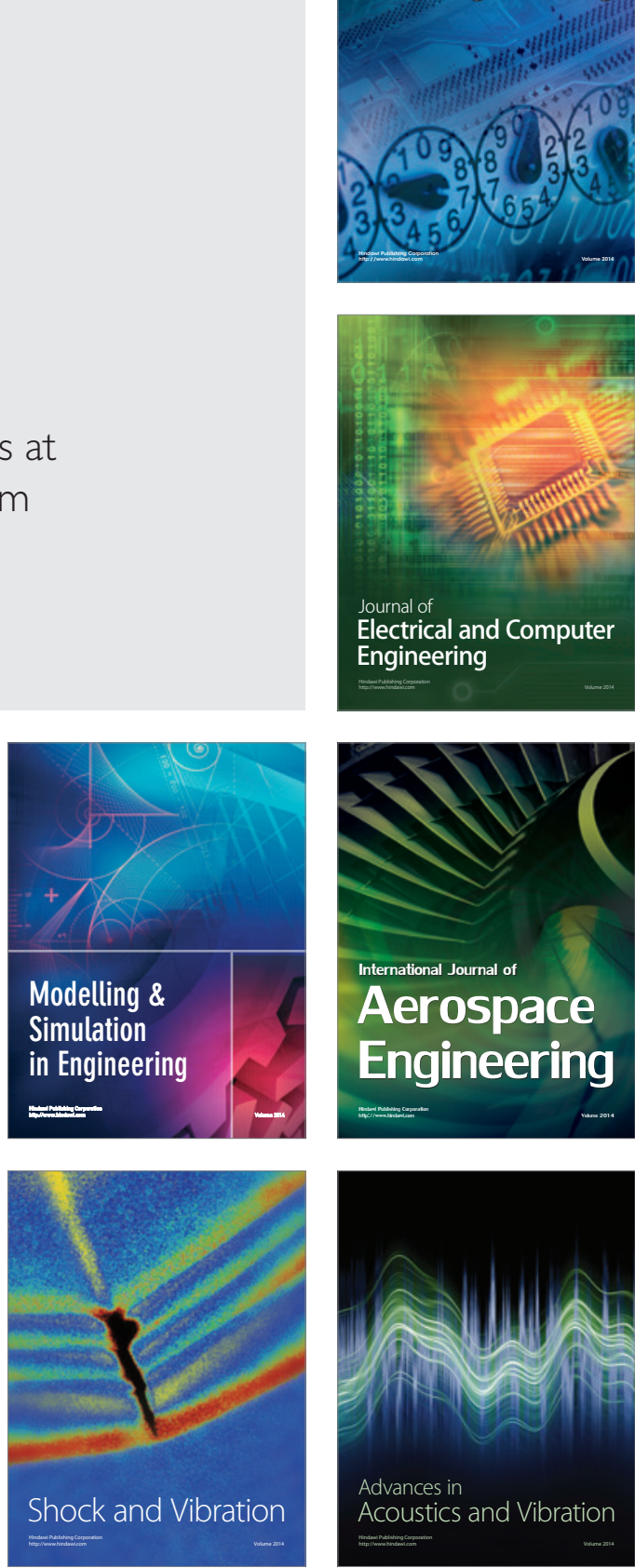